\title{
Closed-loop neuromodulation of spinal sensorimotor circuits controls refined locomotion after complete spinal cord injury
}

Nikolaus Wenger et al.

Sci Transl Med 6, 255ra133 (2014);

DOI: $10.1126 /$ scitranslmed.3008325

Editor's Summary

\section{Closing the Loop on Neuroprosthetic Control}

Patients with spinal cord injury ( $\mathrm{SCl}$ ) and paralysis may soon be outfitted with so-called neuromodulation devices, which electrically stimulate the brain or spinal cord, causing movement in the legs. Currently, tuning such modulation requires constant observation and patient-specific adjustments, which are not ideal for fluid movement or for broad translation of these technologies to injured patients. In response, Wenger et al. have created a closed-loop system that will essentially "auto-tune" the device, allowing the paralyzed patient--or, in their study, the paralyzed rat --to move freely, without worrying about adjusting electrical pulse width, amplitude, or frequency. The authors gave rats complete SCI epidural electrical stimulation and then mapped their leg movements and sensorimotor responses while in a body support system, walking upright (bipedal) on a treadmill, or climbing stairs. From this information, they devised a computational system that integrated feedback and feed-forward models for closed-loop, continuous control of leg movement and, in turn, a more natural locomotion. Closed-loop neuromodulation of spinal circuits could impart fluid motor control and prevent fatigue when rehabilitating patients with SCl.

A complete electronic version of this article and other services, including high-resolution figures, can be found at:

http://stm.sciencemag.org/content/6/255/255ra133.full.html

Supplementary Material can be found in the online version of this article at: http://stm.sciencemag.org/content/suppl/2014/09/22/6.255.255ra133.DC1.html

Related Resources for this article can be found online at:

http://stm.sciencemag.org/content/scitransmed/5/210/210ra155.full.html http://stm.sciencemag.org/content/scitransmed/6/222/222ra19.full.html http://stm.sciencemag.org/content/scitransmed/5/210/210ra154.full.html http://stm.sciencemag.org/content/scitransmed/5/210/210ps15.full.html http://stm.sciencemag.org/content/scitransmed/5/210/210rv2.full.html http://stm.sciencemag.org/content/scitransmed/5/208/208ra146.full.html http://www.sciencemag.org/content/sci/345/6204/1254927.full.html

Information about obtaining reprints of this article or about obtaining permission to reproduce this article in whole or in part can be found at:

http://www.sciencemag.org/about/permissions.dtl 


\title{
Closed-loop neuromodulation of spinal sensorimotor circuits controls refined locomotion after complete spinal cord injury
}

\author{
Nikolaus Wenger, ${ }^{1 *}$ Eduardo Martin Moraud, ${ }^{2,3 *}$ Stanisa Raspopovic, ${ }^{2,4}$ Marco Bonizzato, ${ }^{2}$ \\ Jack DiGiovanna, ${ }^{2}$ Pavel Musienko, ${ }^{1,5}$ Manfred Morari, ${ }^{3}$ Silvestro Micera, ${ }^{2,4 \dagger}$ Grégoire Courtine $^{1+\ddagger}$
}

\begin{abstract}
Neuromodulation of spinal sensorimotor circuits improves motor control in animal models and humans with spinal cord injury. With common neuromodulation devices, electrical stimulation parameters are tuned manually and remain constant during movement. We developed a mechanistic framework to optimize neuromodulation in real time to achieve high-fidelity control of leg kinematics during locomotion in rats. We first uncovered relationships between neuromodulation parameters and recruitment of distinct sensorimotor circuits, resulting in predictive adjustments of leg kinematics. Second, we established a technological platform with embedded control policies that integrated robust movement feedback and feed-forward control loops in real time. These developments allowed us to conceive a neuroprosthetic system that controlled a broad range of foot trajectories during continuous locomotion in paralyzed rats. Animals with complete spinal cord injury performed more than 1000 successive steps without failure, and were able to climb staircases of various heights and lengths with precision and fluidity. Beyond therapeutic potential, these findings provide a conceptual and technical framework to personalize neuromodulation treatments for other neurological disorders.
\end{abstract}

\section{INTRODUCTION}

Regulation of dysfunctional neuronal circuits with electrical neuromodulation therapies has broadened the spectrum of treatment options for neurological disorders $(1,2)$. For example, deep brain stimulation of basal ganglia has become a common medical practice to alleviate motor symptoms of Parkinson's disease (3-6). Experimentally, epidural electrical stimulation (EES) and intraspinal stimulation of the lumbosacral and thoracic spinal segments have enabled improved motor control capabilities in rodents and humans with spinal cord injury (SCI) (7-9) and Parkinson's disease $(10,11)$. In these experiments, empirical knowledge and visual observations guided the tuning of electrical neuromodulation parameters. After manual adjustment of pulse width, amplitude, and frequency, stimulation of the brain and spinal cord remained constant. Such an approach is likely suboptimal to mediate maximum therapeutic effects in patients $(1,2)$. Indeed, preliminary evaluations have suggested that nonregular temporal patterns $(5,6)$ and closed-loop adjustment $(4,12)$ of deep brain stimulation augment the therapeutic impact of neuromodulation.

The ability to modulate the parameters and timing of EES of lumbosacral circuits to improve motor control after SCI remains unclear. Various studies have shown that tuning frequency, amplitude, and pulse width of EES can modulate specific aspects of standing, stepping, and isolated movements in both animal models and humans $(8,13-15)$.

${ }^{1}$ International Paraplegic Foundation Chair in Spinal Cord Repair, Center for Neuroprosthetics and Brain Mind Institute, Swiss Federal Institute of Technology (EPFL), Lausanne $\mathrm{CH}-1015$, Switzerland. ${ }^{2}$ Translational Neural Engineering Lab, Center for Neuroprosthetics and Institute of Bioengineering, School of Bioengineering, Swiss Federal Institute of Technology (EPFL), Lausanne CH-1015, Switzerland. ${ }^{3}$ Automatic Control Laboratory, Swiss Federal Institute of Technology (ETHZ), Zurich CH-8092, Switzerland. ${ }^{4}$ The BioRobotics Institute, Scuola Superiore Sant'Anna, Pisa IT-56025, Italy. ${ }^{5}$ Pavlov Institute of Physiology, St. Petersburg RU-100034, Russia.

*These authors contributed equally to this work.

†These authors contributed equally to this work.

‡Corresponding author. E-mail: gregoire.courtine@epfl.ch
However, to our knowledge, a comprehensive mapping of the functional relationships between stimulation parameters and limb kinematics that could be leveraged to achieve closed-loop control of EES has not been conducted.

Several limitations have restricted the development of closed-loop control systems for neuromodulation (16). First, efficient control policies require a mechanistic understanding of the relationships between stimulation features, the recruitment of specific sensorimotor circuits, and the resulting functional effects. Second, closed-loop control of neuromodulation relies on the availability of a stable and robust feedback signal to tune stimulation parameters. Third, implementation and optimization of closed-loop control algorithms necessitate advanced technological platforms combining feedback and feed-forward loops that match the natural flow of information in the modulated neural systems. Here, we sought to address these issues simultaneously to modulate lumbosacral circuits through closed-loop EES.

Previous studies using EES to improve standing and walking in severely paralyzed subjects have operated under the assumption that the stimulation primarily increases the general level of excitability within spinal circuits (8) and activates central pattern generators (13). However, EES is also able to recruit specific afferent pathways connected to distinct sensorimotor circuits, which, in turn, generate welldefined responses in leg muscles after each pulse of stimulation (17-19). We first capitalized on this understanding of EES mechanisms to establish a mapping between EES parameters, neuromodulation of sensorimotor circuits, and adjustments of leg movements. Second, we developed robust algorithms to extract a useful feedback signal from spatiotemporal characteristics of leg kinematic during locomotion. Third, we established a versatile technological platform with embedded control policies that interfaced feedback signals and feed-forward models operating in real time. These integrated developments allowed us to conceive a closed-loop neuromodulation system that controlled a broad range of leg movements during natural locomotion in paralyzed rats. 


\section{RESULTS}

\section{Modulation of EES parameters tunes specific aspects of leg movements}

We first characterized the impact of EES amplitude, pulse width, and frequency on the modulation of leg kinematic, ground reaction force, and muscle activity in adult rats that received a complete transection of the mid-thoracic spinal cord (fig. S1). After 5 weeks of recovery, all of the tested rats $(n=5)$ dragged both hindlimbs along the treadmill belt when supported bipedally in a robotic body weight support system (Fig. 1A). The combination of serotonin agonists $\left(5 \mathrm{HT}_{1 \mathrm{~A} / 2 / 7}\right)$ and EES delivered at S1 and L2 spinal segments (Fig. 1B) promoted continuous and alternating stepping movements of the legs. Frequency, amplitude, and pulse width of EES were adjusted to cover the entire range of values that promoted functional movements.

Increments of EES frequency led to a graded and consistent modulation of gait patterns in all the tested rats within a range of 20 to $90 \mathrm{~Hz}$ (Fig. 1, C and D). To characterize this modulation and identify the most relevant features reflecting tuning of leg movements with EES frequency, we computed 147 kinematic, force, and electromyographic (EMG) variables that provided a comprehensive description of the locomotor state (listed in table S1). We subjected these combined parameters to a PC analysis. Stepping patterns associated with each EES frequency were differentiated along PC1, which explained nearly $50 \%$ of the total variance in the data sets (Fig. 1D). Analysis of factor loadings on PC1 revealed that step height was the more robust variable (factor loading, 0.97 ) to capture the modulation of leg movements during changes in EES frequency (fig. S2). There were linear correlations between EES frequency and step height that were reproducible within a session (Fig. 2A), over weeks (Fig. 2A), and among rats (Fig. 2B), despite idiosyncratic gait patterns. Tuning EES frequency over the useful range of values (shaded areas in Fig. 2A) mediated a threefold increase in foot elevation, which allowed rats to cover step heights ranging from $2.9-$ to $6.8-\mathrm{cm}$ mean across rats.

Increase in EES amplitude (Fig. 3, A to C) mediated adjustments of lower limb kinematics similar to those observed with changes in EES frequency, including a significant, albeit limited increase in step height $(2.8$ to $3.9 \mathrm{~cm}$ ) (Fig. 3C). Changes in limb kinematics were less systematic when adjusting pulse width (Fig. 3, D to F). Moreover, increase in EES amplitude and pulse width induced coactivation of leg muscles (Fig. 3, A and D), which led to a rapid saturation of stepping movements, and thus limited the relevance of these parameters for neuromodulation strategies.

\section{EES frequency modulates specific sensorimotor circuits}

We next sought to identify the physiological principles underlying the modulation of step height with EES frequency. EES primarily recruits proprioceptive afferent fibers $(17,19,20)$. Here, we found that the recruitment of proprioceptive fibers engages monosynaptic and polysynaptic reflex circuits, which elicited well-defined motor responses in leg muscles after each pulse of EES (Fig. 4A). We dissociated these responses functionally based on their respective latencies (mediumand late-latency responses). In turn, phase-dependent modulation of these responses in flexor versus extensor muscles resulted in alternating bursts of EMG activity, enabling continuous locomotion in otherwise paralyzed rats (Fig. $4 \mathrm{~B}$ and fig. S3, A and B).

EES-induced motor responses remained locked to each pulse of stimulation across the entire range of tested EES frequencies (Fig. 4,
A

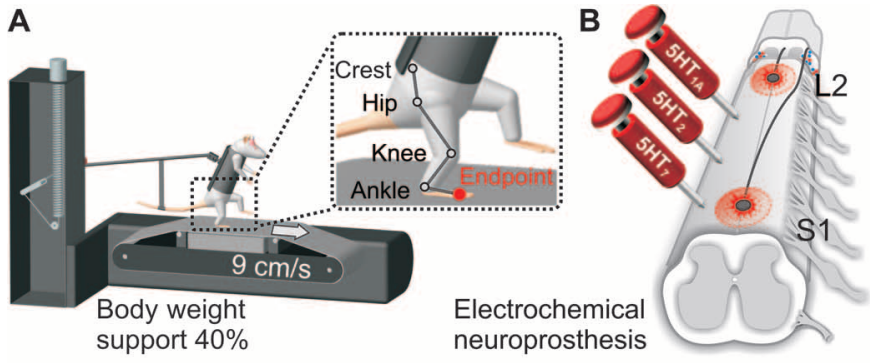

C

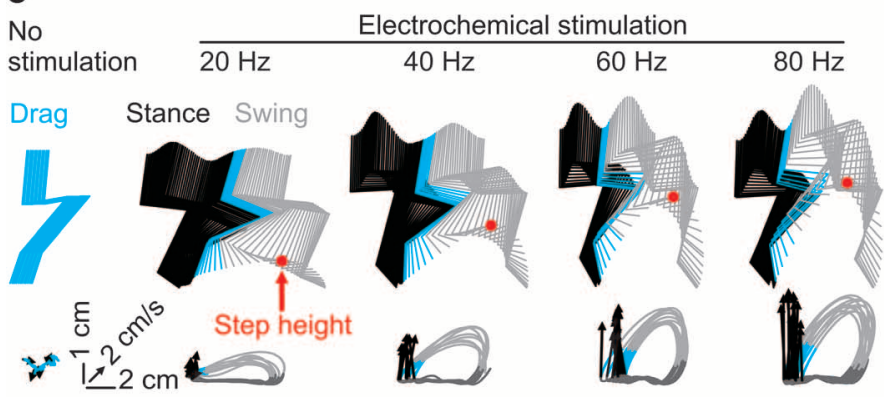

Endpoint trajectory $n=10$ steps
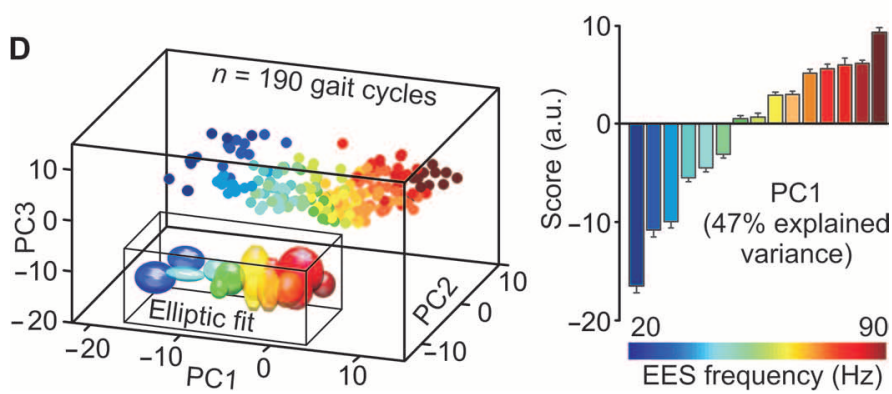

Fig. 1. Modulation of EES frequency tune foot trajectory during locomotion. (A) Rats with complete $\mathrm{SCl}$ were positioned bipedally over a treadmill belt using an adjustable body weight support system. In the inset, markers were positioned over leg joints, including the limb endpoint. (B) EES was applied along the midline of L2 and S1 spinal segments. Serotonin agonists (5HT) were administered systemically before stepping. (C) Representative, color-coded stick diagram decomposition of hindlimb movements during stance (black), drag (cyan), and swing (gray), together with 10 successive trajectories of the limb endpoint. The red dot indicates the maximum step height. The black arrows represent the direction and intensity of the limb endpoint trajectory at swing onset. (D) Principal component (PC) analysis applied on all 147 gait variables (table S1) measured over 10 successive gait cycles during EES frequencies ranging from 20 to $90 \mathrm{~Hz}$, with a $5-\mathrm{Hz}$ increment for a representative rat. Least-square elliptic fits are drawn to emphasize frequency-dependent modulation of gait patterns. The bar graph reports the score for each EES frequency along PC1. Data are means + SEM. a.u., arbitrary units.

$\mathrm{B}$ and C). Consequently, increment of EES frequency induced a graded increase in the number of medium- and late-latency responses in leg muscles (Fig. 4, C and D, and fig. S3C). At the lower frequencies, both responses independently contributed to the elaboration of muscle activity in both flexor and extensor muscles (fig. S3D). At higher frequencies, the monosynaptic input likely reached motoneurons at the same time as the polysynaptic input from the preceding stimulation. Temporal convergence of monosynaptic and polysynaptic inputs onto 

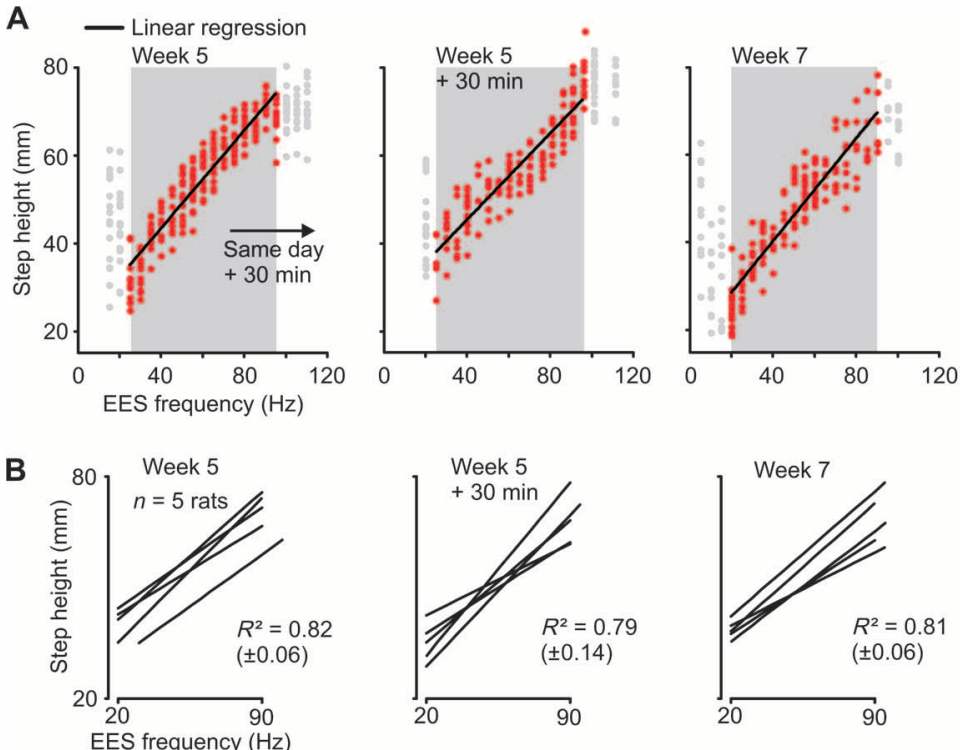

Fig. 2. Reproductive modulation of step height with EES frequency within and across days, and between rats. (A) Relationship between EES frequency and step height for a representative rat tested 30 min apart in the same session 5 weeks post-lesion, and at 7 and 9 weeks post-lesion. The linear regression (back line) was computed over the range of functional EES frequencies (red dots, shaded areas). Each dot represents a gait cycle. (B) Plots reporting the linear regressions from five rats recorded in the same conditions as in (A).

motoneurons eventually led to a single, medium-latency response, which accounted for the increase in the amplitude of this response with larger EES frequencies (fig. S3, C and D).

The graded increase in the number and amplitude of motor responses led to a linear enhancement of the overall bilateral EMG activity in extensor and flexor ankle muscles (Fig. 4E and fig. S3D). In turn, the concurrent modulation of ankle extensors in the stance leg and flexor muscles in the swing leg accounted for the progressive tuning of step height when adjusting EES frequency (Fig. 4E). These results indicate that tuning of EES frequency mediates a progressive and reproducible modulation of monosynaptic and polysynaptic reflex circuits, which promotes predictive adjustment of leg movements during locomotion in rats.

\section{Closed-loop EES achieves high-fidelity control of leg movements}

Linear tuning of step height with increase in EES frequency opened the intriguing possibility to automate leg movement control in real time through closed-loop modulation of EES frequency. To test this hypothesis, we designed a technological platform that combined (i) online monitoring of kinematics, ground reaction force, and muscle activity in real time; (ii) signal-processing algorithms to reconstruct locomotor states, including limb endpoint trajectory, gait phases, and whole-body position; and (iii) a computational infrastructure to embed control policies in the feedback loop to adjust EES frequency based on current and desired stepping behaviors (Fig. 5A, fig. S4, and movie S1). Optimization of communication protocols and informationprocessing time allowed the entire loop to operate in less than $20 \mathrm{~ms}$ (see Materials and Methods), which was appropriate for real-time control of neuromodulation during gait despite the fast rhythm of stepping in rats (cycle duration, $810 \pm 156 \mathrm{~ms}$, SD).
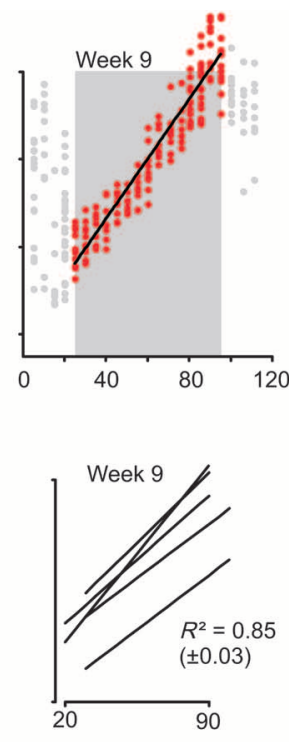

We used this real-time platform to implement a closed-loop control policy capable of adjusting step height in real time during locomotion. The continuous flow of kinematic information was discretized per gait cycle and merged into a single variable (step height) that was used to automatically adjust EES frequency during the next gait cycle (Fig. 5A). The controller adjusted stimulation to maintain the maximum elevation of the foot within a reference band, whose upper and lower bounds $( \pm 5 \mathrm{~mm}$ ) were selected to account for the intrinsic variability of step height during continuous locomotion $(4.6 \pm 0.9 \mathrm{~mm}$, $\mathrm{SD}$ ). This single-input single-output (SISO) control structure integrated parallel loops combining adaptive feed-forward prediction and feedback correction (Fig. 5A and fig. S4).

The feed-forward model was derived from the linear correlation between step height and EES frequency recorded for each rat at the beginning of each session (Fig. 2). The slope and intercept values of the model were iteratively updated after each gait cycle to account for time-varying changes in stepping behavior. A proportional-integral (PI) feedback loop complemented the feed-forward model predictions to minimize the discrepancy between the desired and actual step height (movie S1). This architecture achieved a high degree of control over a broad range of step heights during both steady and constantly changing locomotor states (Fig. 5, B and C).

To quantify the degree of step height control, we designed a comprehensive suite of testing paradigms, in which the reference band was displaced through a range of fixed increments or decrements after each step, or after a series of steps (Fig. 5C). These tasks tested the performances of the controller during steady-state (fig. S5A) and dynamic (fig. S5, B and C) behaviors over the entire range of step height modulations. Despite these multifaceted challenges, the controller successfully tuned EES frequency to instantly adjust step height to varying reference bands (movie S2). Precision of step height control significantly increased in variability (fig. S5D) when imposing adjustments that reached the physiological limits of the studied biomechanical system.

\section{Closed-loop EES prevents rapid fatigue during continuous locomotion}

We next sought to evaluate the ability of closed-loop EES to improve key aspects of gait performance. Human patients with SCI exhibit a rapid exhaustion of locomotor output during continuous stepping on a treadmill (21), which is also observed in injured rats (22). During noncontrolled EES $(40 \mathrm{~Hz})$, rats with complete SCI showed a progressive decrease in the amplitude of muscle activity during stepping (Fig. 6, A and B), which led to a graded decrease in step height until the animals collapsed (Fig. 6C).

Instead, closed-loop control of step height doubled the duration of stepping before collapse compared to noncontrolled EES (Fig. 6C). The controller continuously increased EES frequency over time, which limited the decrease in the amplitude of muscle activity (Fig. 6B), and 
A EES amplitude modulation

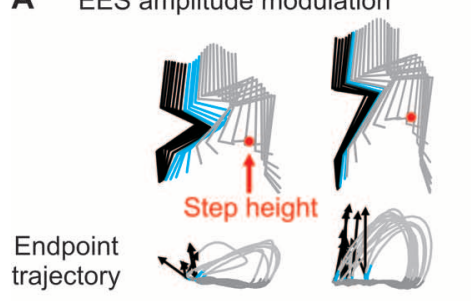

Stance Swing Drag
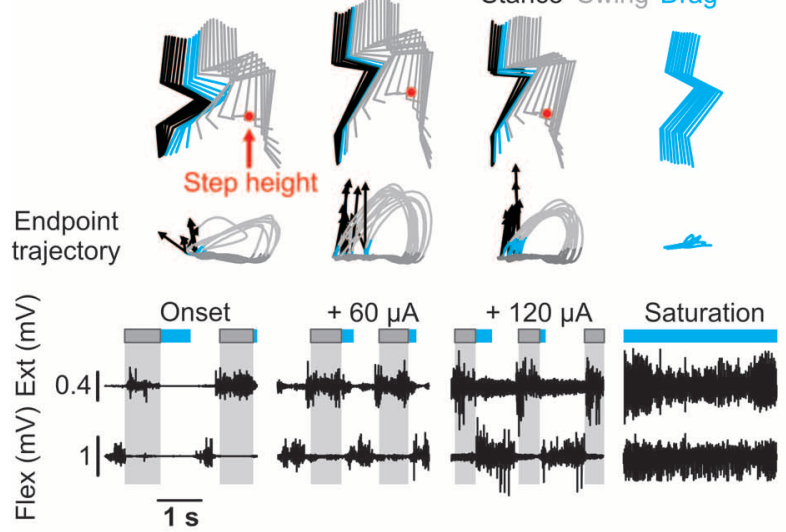

D EES pulse width modulation

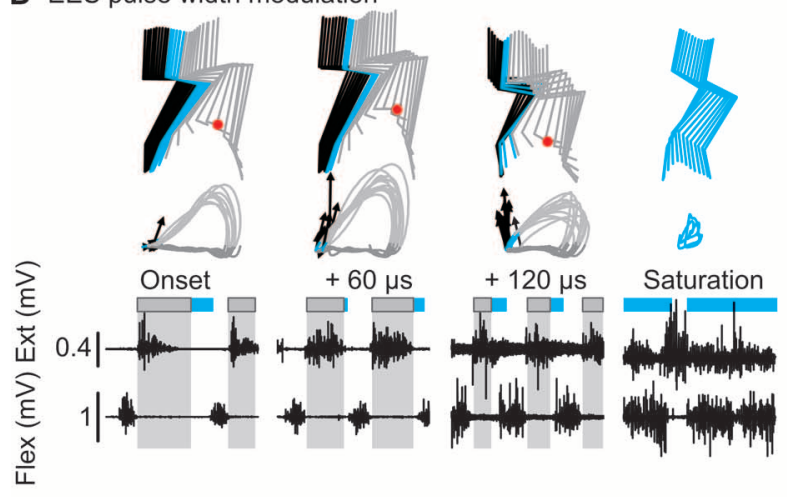

Fig. 3. EES amplitude and pulse width mediate limited tuning of leg movements during locomotion. (A) EES amplitude was gradually increased by fixed increments of $60 \mu \mathrm{A}$ from the onset of EES-induced stepping, until saturation. EES frequency was kept constant at $40 \mathrm{~Hz}$. Stick diagram decomposition of hindlimb movements, successive limb endpoint trajectories, and alternating flexor (Flex) and extensor (Ext) EMG activity are displayed for each experimental condition using the same convention as in Fig. 1. (B) PC analysis was applied on all the gait variables measured in a representative rat during EES amplitude changes shown in (A). The bar

enabled rats to maintain step height within the constant reference band for extended durations despite exhaustion (Fig. 6C and movie S3). Under controlled conditions, $100 \%$ of the tested rats $(n=3)$ performed at least 1000 successive steps without failure over the duration of the testing session.

\section{Closed-loop EES enables locomotion across combinations of staircases}

We lastly aimed to illustrate the practical impact of closed-loop EES to restore walking during more natural locomotor conditions. For this, we positioned the rats overground in a robotic interface that moved the rats forward along a runway while providing constant-force support in the vertical (25\% of body weight) and mediolateral (rigid, $100 \%$ ) directions (Fig. 7A). We interposed staircases of various heights (1.3 to $3.5 \mathrm{~cm}$ ), lengths ( 12 to $25 \mathrm{~cm}$ ), and numbers (one to four stairs) along the runway to test the ability of closed-loop EES to modulate step height to accommodate leg movements during stair climbing. The reference band was set at a constant height during locomotion along the horizontal surface until crossing a predefined distance from
B
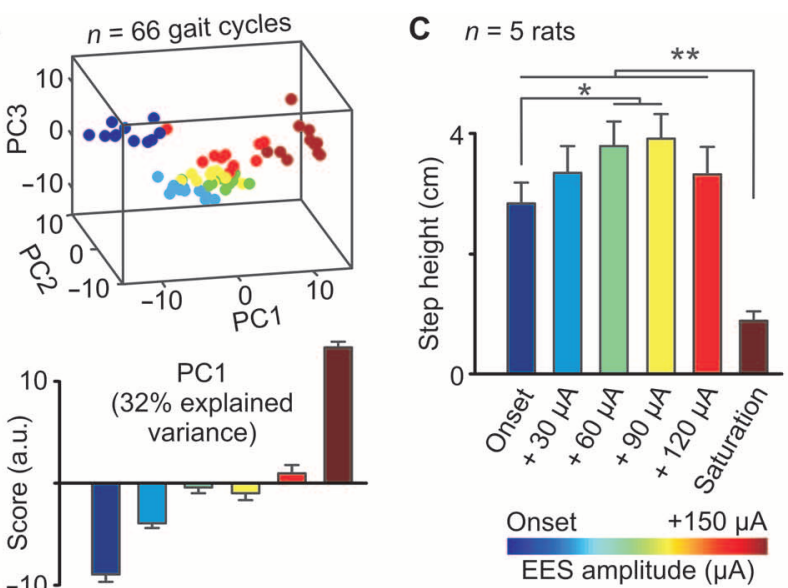

$\mathbf{E}^{-10}$
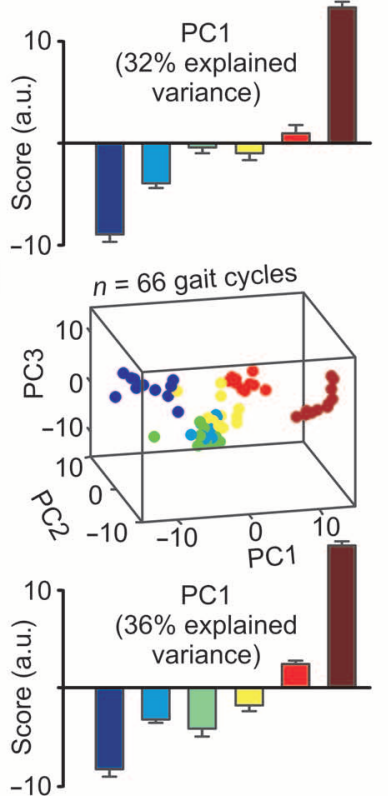

$\mathbf{F}$

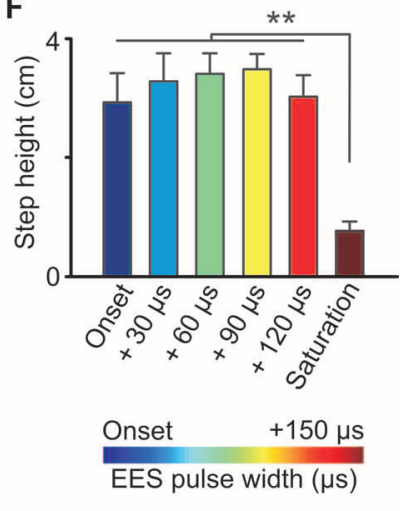

graph reports the score for each EES amplitude along PC1 for all tested rats $(n=5)$. (C) Histogram plots reporting changes in step height in response to modulation of EES amplitude. (D) EES pulse width was gradually increased by fixed increments of $60 \mu \mathrm{s}$ in the same rat and under the same conditions as (A). (E) PC analysis was applied on all the gait variables measured during EES pulse width changes shown in (D). (F) Histogram plots reporting changes in step height in response to modulation of EES pulse width. ${ }^{*} P<0.05,{ }^{* *} P<0.01$, repeated-measures analysis of variance (ANOVA) with Bonferroni post hoc analysis. Data are means \pm SEM $(n=5)$.

the staircase $(1.5 \mathrm{~cm})$ where the reference band was raised to an appropriate height $(3 \mathrm{~cm}$ above staircase height) to pass the staircase. During noncontrolled EES, rats tumbled against and failed to pass the lower staircase (Fig. 7A). In contrast, closed-loop EES enabled the rats $(n=3)$ to climb all of the tested staircase combinations successfully in 99 of 100 successive attempts $(P<0.001$ versus noncontrolled EES, Kruskal-Wallis test) (Fig. 7, A and B, and movie S4).

Automated tuning of EES frequency during the stance phase preceding the staircase significantly augmented vertical ground reaction forces proportional to the displacement of the reference band (Fig. 7B), which generated the adequate foot trajectory to overcome the staircase. The same precision of step height control was obtained during sequences of staircases, which required a continuous adjustment of EES frequency to pass the successive stairs while stabilizing upward and downward movements of the body after each step (fig. S6). These combined results show the ability of the developed neuromodulation algorithms to achieve real-time adjustment of leg kinematic and ground reaction forces with adequate precision to restore complex walking behaviors in paralyzed rats. 
A Standing

Stimulation
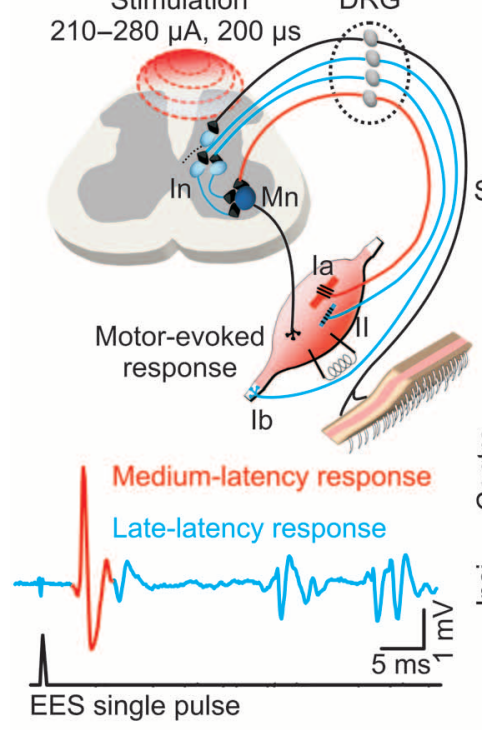

C FFT
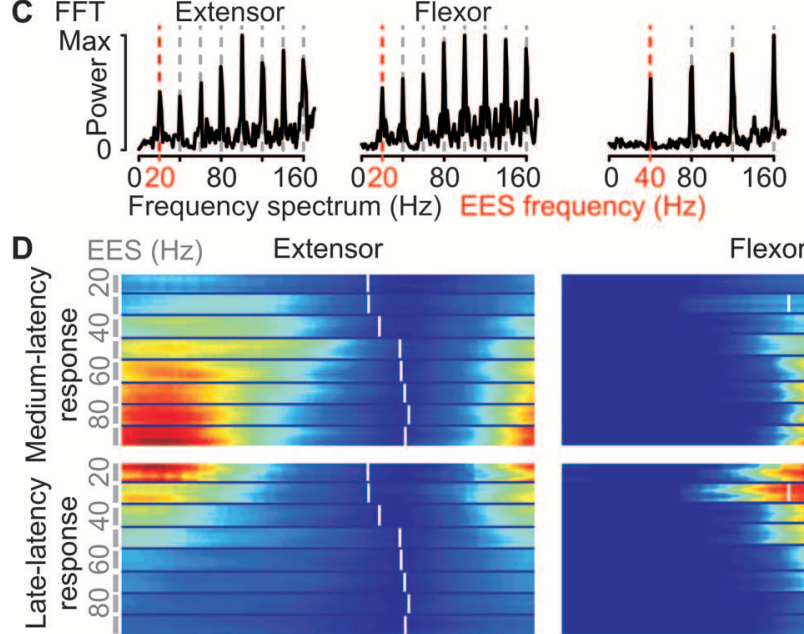

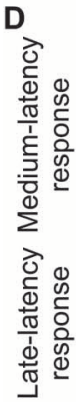

B Stepping

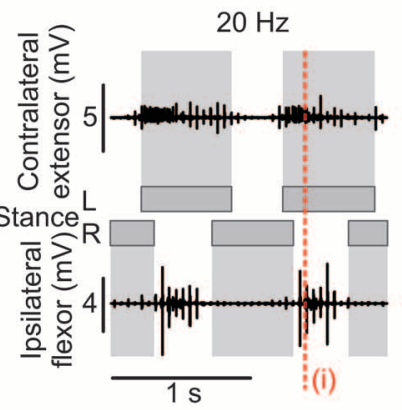

(i)

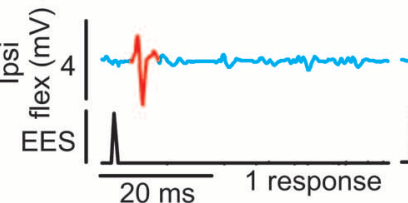

Flexor
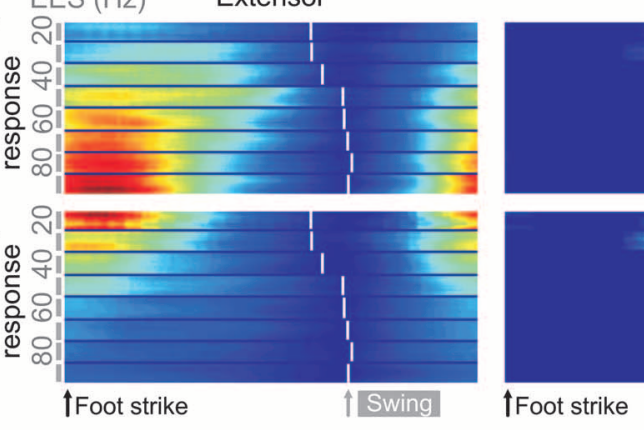
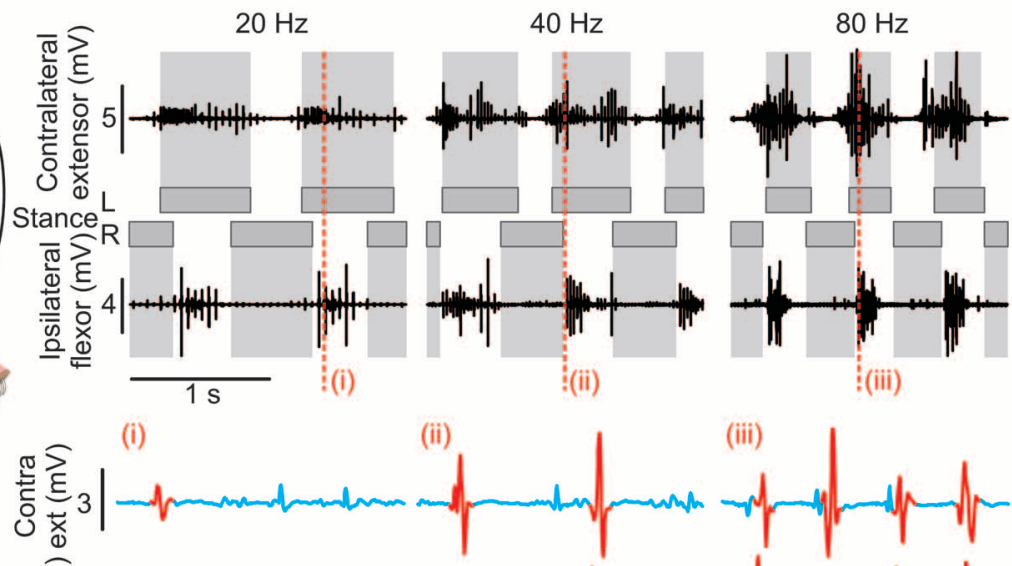

(iii)
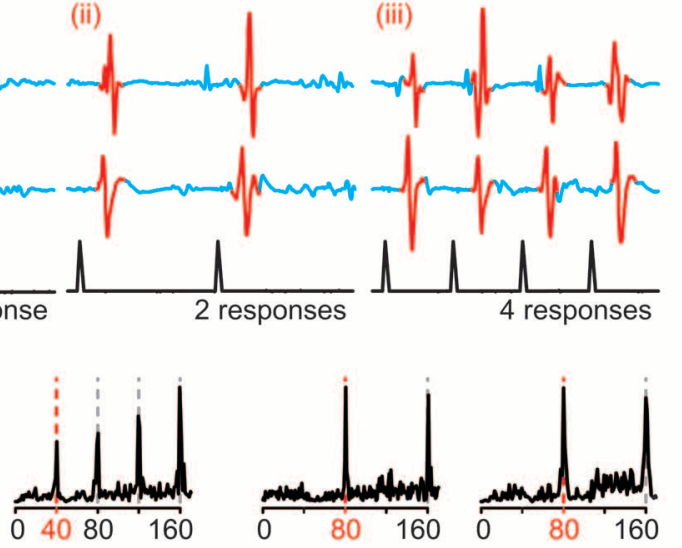

100 response activity (\%)

Fig. 4. EES frequency modulates specific reflex circuits. (A) Schematic of neural structures recruited by EES. In, interneuron; Mn, motoneuron; DRG, dorsal root ganglion; lb, group lb fibers conveying force feedback; la and II, group la and group II fibers conveying muscle length-related feedback. A single pulse of EES evokes a medium-latency response (red) and a late-latency response (blue) in leg muscles through activation of proprioceptive segmental circuits. (B) Sequence of EMG activity recorded in the contralateral extensor (medial gastrocnemius) and ipsilateral flexor (tibialis anterior) muscles during continuous stepping at three different EES frequencies. The lower traces show, for each frequency, motor responses evoked in extensor (ext) and flexor (flex) muscles at distinct moments of the gait cycle (i, ii, and iii), but over the same duration. (C) Power spectrum

\section{DISCUSSION}

Open-loop regulation of spinal sensorimotor circuits with neuromodulation therapy improves motor control after SCI $(7,8,14,23)$. Here, we demonstrate that closed-loop neuromodulation yielded superior control of leg movement compared to continuous stimulation, enabling rats

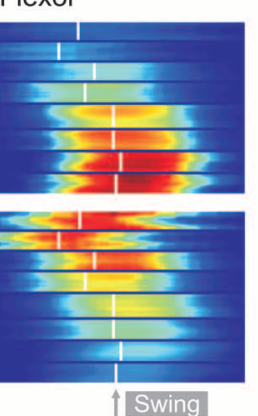

20

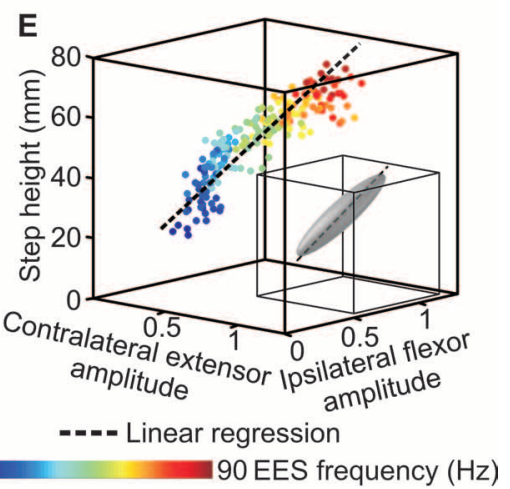

of EMG bursts for flexor and extensor muscles at different stimulation frequencies. The red and gray vertical lines indicate EES frequency and its harmonic multiples, respectively. (D) Color-coded modulation of the amplitude of medium- and late-latency responses in flexor and extensor muscles over the course of a gait cycle for EES frequencies ranging from 20 to $90 \mathrm{~Hz}$, with a $10-\mathrm{Hz}$ increment. The white lines indicate the end of stance for each frequency. (E) Three-dimensional (3D) linear correlation between colorcoded EES frequency and normalized EMG amplitude of contralateral extensor and ipsilateral flexor muscles. Each point represents an individual gait cycle. The robustness of the correlation was calculated through PC analysis as the amount of explained variance by PC1. All panels report data recorded in the same representative rat (representative of $n=5$ ).

with complete SCI to perform complex locomotor tasks with precision and fluidity. Although the current demonstration is in rodents, research platforms for human spinal cord stimulation (16) and a newly developed robotic interface (24) provide the technological infrastructure to achieve immediate translation of our control algorithms into neuromodulation therapies to facilitate robot-assisted training in humans with SCI. 
A

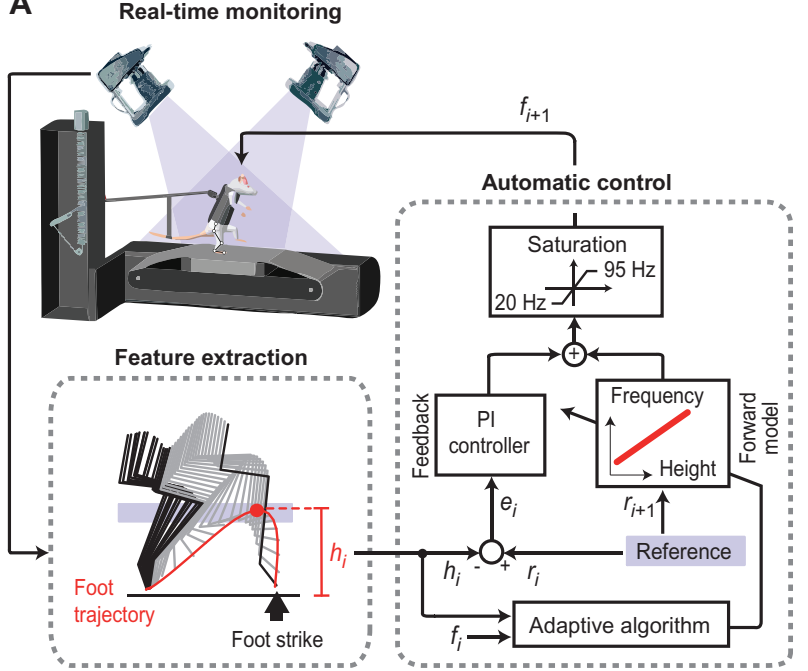

B

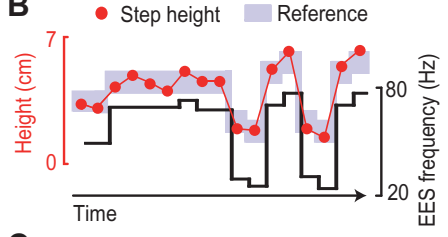

C

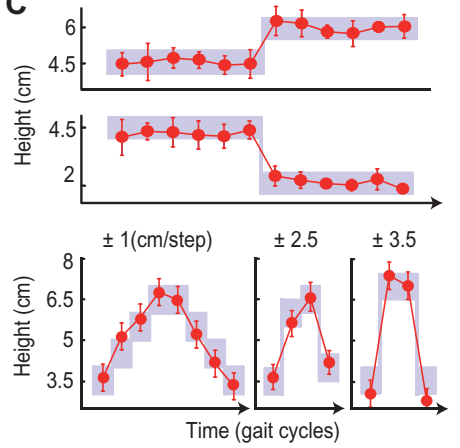

Fig. 5. Real-time control of step height during locomotion. (A) The architecture of the closed-loop system combined real-time monitoring of kinematic information and automated control policies. After each foot strike, the signal-processing algorithm measured the step height (red dot, $\left.h_{i}\right)$, which was fed into the control loop. The control structure integrated parallel loops combining feedback correction (PI controller) and a predictive forward model. This predictive component was elaborated from the linear relationship between EES frequency and step height, and was updated at each iteration through an adaptive algorithm (see Materials and Methods). The controller combined the error $\left(e_{i}\right)$ correction based on the current reference $\left(r_{i}\right)$, and the prediction to generate the adjustment of EES frequency $\left(f_{i+1}\right)$ to adjust the current frequency $\left(f_{i}\right)$ and thus maintain the maximum step height within the user-defined reference band $\left(r_{i+1}\right.$, shaded areas). A saturation function was inserted to constrain EES within the functional range of frequencies. (B) Example of successive step heights and concomitant EES frequencies during a random sequence of reference band changes for one rat. (C) Mean values \pm SEM ( $n=15$ trials) of successive step heights for various combinations of steady and constant-changing reference bands (shaded area).

EES of lumbosacral circuits facilitates locomotion through the recruitment of proprioceptive afferent fibers, which engages two complementary mechanisms (17). First, the propagation of the tonic neural drive elicited along myelinated afferent fibers activates central patterngenerating networks $(25,26)$. Second, each stimulation pulse elicits a medium- and late-latency response in leg muscles, which corresponds to the activation of monosynaptic and polysynaptic reflex circuits, respectively $(13,17,19)$. Central pattern-generating networks modulate the gain of these reflex circuits according to the state of the locomotor system $(19,27)$. In turn, the successive motor responses compose the bursts of leg muscle activity during locomotion $(17,19)$. Here, we found that spinally evoked motor responses remain locked to the stimulation rate over a large range of frequencies, enabling fine-tuning of muscle activity through linear adjustment of stimulation. Likewise, frequencydependent modulation of intraspinal microstimulation delivered in cervical segments near-linearly adjusts the amount of grasp force in anesthetized nonhuman primates (28). This principle underlies natural muscle force production, which primarily relies on the modulation of motoneuron firing rate (29).

We leveraged this mechanistic framework to achieve real-time control of lower limb kinematics and vertical ground reaction forces during continuous locomotion. This control policy allowed paralyzed rats to produce a broad range of foot trajectories during continuous locomotion, and to climb combinations of staircases. Frequency-dependent tuning functions were consistent across days and across rats, which supported the design of algorithms with robust performances. This treatsimple control policy onto the ongoing motor program to achieve continuous, high-fidelity control of leg movements over extended periods of time.

Our control policy combined feedback signals and feed-forward models that relied on a global parameter capturing the targeted locomotor behavior. For this purpose, we extracted spatiotemporal features from lower limb endpoint trajectories. There is evidence that central nervous system circuits may elaborate an explicit representation of limb endpoint kinematics (35). Moreover, neurobehavioral experiments have suggested that the limb endpoint is the primary variable used to coordinate locomotion in animal models (36) and humans (37). We thus monitored and controlled step height, which was the most robust parameter to account for frequency-dependent modulation of gait. In addition, lower limb endpoint is a neurally relevant variable to encode and organize leg movements. This type of control policy establishes a framework to design a corticospinal neuroprosthesis through which neuronal modulations from the motor cortex will directly modulate electrical spinal cord stimulation to adjust lower limb kinematics and improve locomotor recovery (38). Current wearable and implantable motion sensors can generate the necessary feedback to incorporate automated closed-loop control policies into neuromodulation therapies for use outside laboratory environments (39). For this purpose, industrial development of clinically viable neuromodulation platforms has established the appropriate neurotechnology to personalize stimulation algorithms based on feedback signals (16). 
A

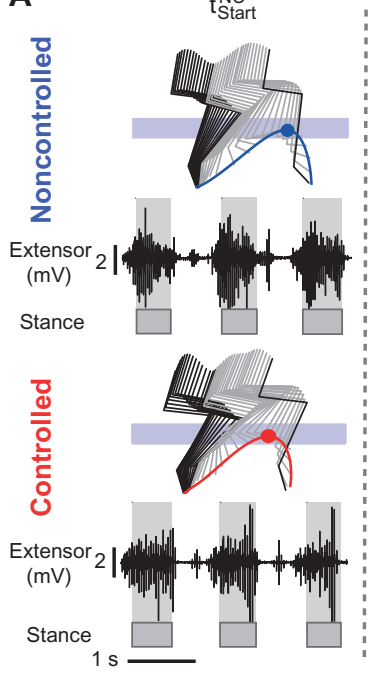

B $(n=3)$

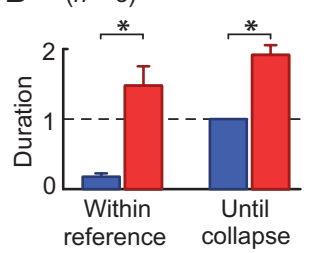

C$$
\text { C }
$$

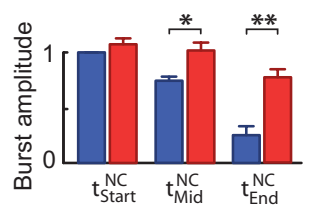

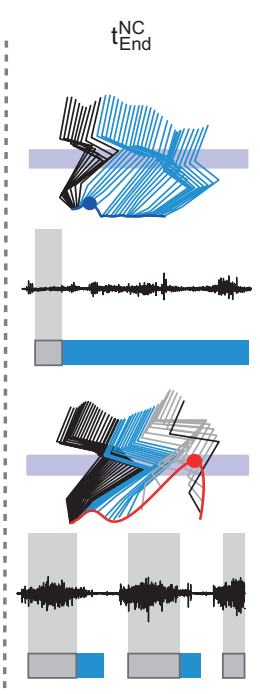

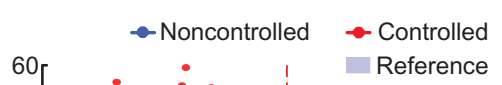

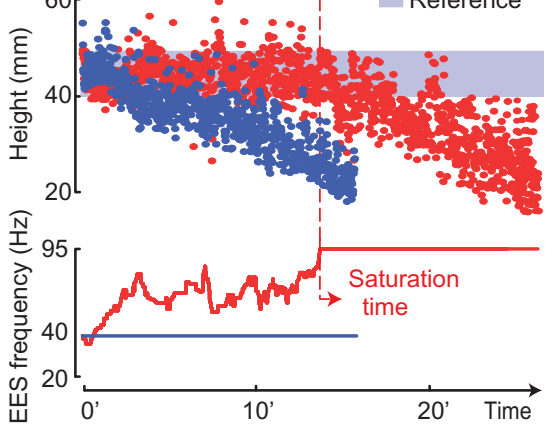

Fig. 6. Closed-loop EES compensates for exhaustion. (A) Stick diagram decomposition of hindlimb movements and extensor muscle activity for both noncontrolled and controlled conditions at three different time points: beginning, middle, and end of the noncontrolled session. Conventions are the same as in Fig. 1C. The gray- and cyan-shaded areas indicate the duration of the stance and drag phases, respectively. The reference (gray band) was set on the basis of the average step height measured across rats. (B) Histogram plots reporting the durations of stepping until collapse and within the reference band, and the amplitude of extensor muscle activity (medial gastrocnemius, MG) for the three time points shown in (A) during noncontrolled and controlled conditions for all the rats ( $n=3$ rats). (C) Representative examples of successive step heights and EES frequency modulation during a continuous sequence of stepping under noncontrolled and controlled conditions. The vertical dotted line corresponds to the saturation time when EES frequency reached the maximum allowed value for the controller, that is, $95 \mathrm{~Hz}$ (Fig. 5A). Each data point corresponds to a gait cycle. Data are means \pm SEM. ${ }^{*} P<0.05,{ }^{* *} P<0.01$, Kruskal-Wallis test.

Current knowledge of human spinal cord properties in response to electrical neuromodulation $(23,31)$ suggests that the control policies developed in our study could translate into clinical applications for facilitating neurorehabilitation $(8,23)$. Existing technological platforms for closed-loop neuromodulation of the human nervous system (16) can readily be interfaced with real-time kinematic feedback to tune EES in clinical settings. Moreover, our results in rats (40) compelled the development of a robotic body weight support system that can provide patients with adjustable, multidirectional support during overground

A
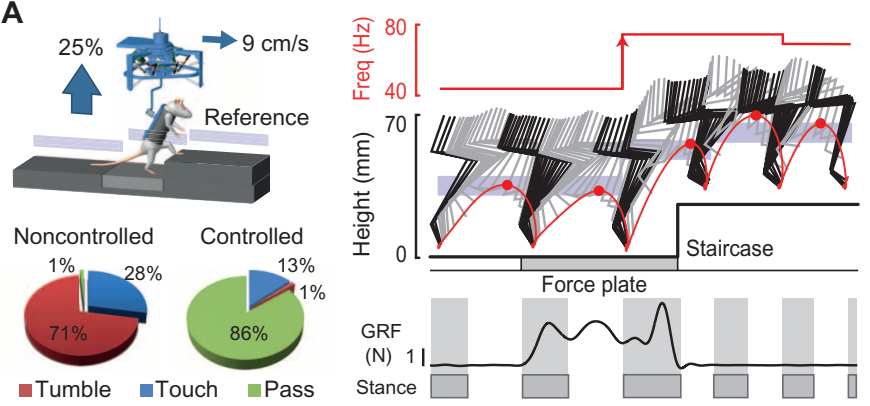

B $\quad(n=3)$
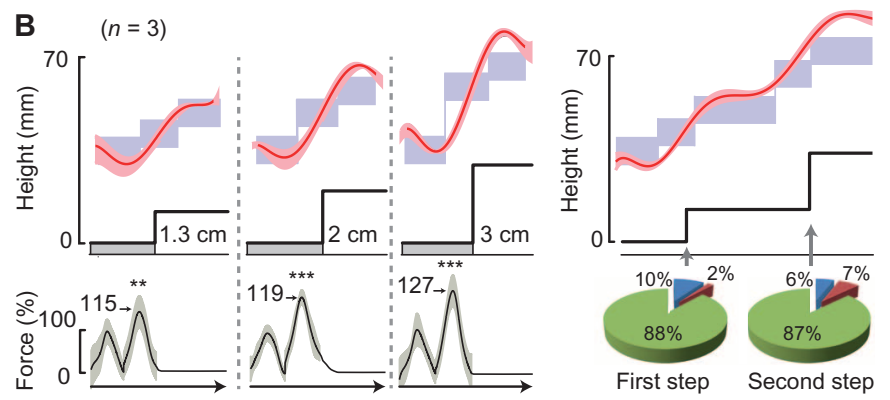

First step Second step

locomotion within a large workspace (24). Thus, our developed algorithms can be implemented to facilitate rehabilitation during robotassisted locomotion on a treadmill and overground in patients with SCI. Closed-loop neuromodulation of spinal circuits has the potential to improve motor control and prevent rapid fatigue during robotassisted training in individuals with incomplete SCI, such as ASIA-C (American Spinal Injury Association grade C) patients, which are essential factors to promote activity-dependent plasticity and recovery with rehabilitation $(8,41)$.

In rats with complete SCI, continuous weight-bearing stepping only emerged in the presence of agonists to specific serotonergic receptor subtypes. Monoaminergic replacement therapies are necessary to mediate locomotor permissive states of spinal circuits deprived of any source of modulation (42). Consequently, closed-loop neuromodulation therapies in humans with severe injuries will be contingent on the development of safe and efficacious monoaminergic replacement strategies, 
which are under exploration (43), but do not yet exist for general use in people. However, open-loop electrical neuromodulation of spinal circuits instantly restored fine control over joint-specific movements in patients with chronic motor paralysis (23). These results suggest that the therapeutic effects of neuromodulation therapies that we demonstrated in rodent models (7) also applied in humans with severe SCI.

Neuromodulation therapies of the brain and spinal cord target distinct neural structures, but share common principles, including the growing need for closed-loop regulation $(1,2,44)$. Our results provide a conceptual and technical framework for the broad development of closed-loop neuromodulation therapies to improve function after neurological disorders.

\section{MATERIALS AND METHODS}

\section{Study design}

The study was segregated into two experimental groups. Characterization of relationships between EES frequency and gait pattern modulation was conducted on a group of five rats that participated in all of the testing sessions over a period of 3 weeks, starting 5 weeks postlesion. After comprehensive analysis of EES characterization and neuromodulation algorithm development, we generated an additional group of three rats that were tested daily over a period of 4 weeks, starting 5 weeks post-lesion. All experiments involving real-time control of EES were carried out with those three rats. This sample size was selected on the basis of the robustness of EES modulation effects and to obtain enough statistical power to demonstrate significant differences between closed- and open-loop neuromodulation. All the tested rats are presented in the study. No statistical outliners were excluded. One animal from the second group had to be excluded from PC analysis owing to a mechanical failure in one of the four EMG wires; however, this rat could be included for analysis in all other figures where unilateral EMG signals were sufficient for data analysis. All measurements were obtained using objective readouts with high-precision equipment. Blinding during data acquisition and analysis was not possible because of the obvious effects of EES modulation.

\section{Animals and animal care}

All procedures and surgeries were approved by the Veterinarian Office Vaud, Switzerland. The experiments were conducted on adult female Lewis rats ( $200 \mathrm{~g}$ of body weight, Centre d'Elevage R. Janvier). Rats were housed individually on a 12-hour light-dark cycle, with access to food and water ad libitum.

\section{Surgical procedure and post-surgical care}

All surgical procedures have been described in detail previously $(7,40)$ and are elaborated in the Supplementary Methods

\section{Locomotor training}

Rats were attached to an automated, servo-controlled body weight support system (Robomedica), and positioned over a motorized treadmill belt for bipedal locomotion. The animals were trained every other day, 20 min per session for 4 weeks, starting 8 days post-SCI. Locomotor training was enabled by the combination of EES at S1 and L2, and systemic administration of agonists to $5 \mathrm{HT}_{1 \mathrm{~A} / 7}(8-\mathrm{OH}-\mathrm{DPAT}, 0.05$ to $0.1 \mathrm{mg} / \mathrm{kg}$ body weight) and $5 \mathrm{HT}_{2 \mathrm{~A} / \mathrm{C}}$ (quipazine, 0.2 to $0.3 \mathrm{mg} / \mathrm{kg}$ body weight) receptor subtypes (42). Treadmill belt speed was set at $9 \mathrm{~cm} / \mathrm{s}$.
Kinematic, ground reaction force, reflex analysis, and EMG recording procedures as well as data and PC analyses are described in the Supplementary Materials.

\section{Real-time platform for closed-loop adjustment of EES}

The platform was implemented within a multithreaded C++ code (Visual Studio 2010, Microsoft) running on a quad core Microsoft Windows 7 computer. Stimulation patterns were applied via an RZ5 processing unit (Tucker-Davis Technologies) connected to an MS16 Stimulus Isolator (Tucker-Davis Technologies). The integrated Vicon recording system generated raw $3 \mathrm{D}$ positions of the markers, which were imported into the C++ environment in (soft) real time via Ethernet using the DataStream SDK software. We developed a custom algorithm for online interpolation of missing markers through triangulation and relabeling of each marker to the appropriate joint landmark. All signals were filtered online using least mean squares adaptive filters. Specific gait events such as foot strike, toe-off, or predetermined timings during each gait cycle were automatically detected using online kinematic classification of limb endpoint trajectories. These timings triggered controller calculations and model updates (fig. S3). To evaluate the delay of the entire platform, we sent concomitant pulses at opposite ends of the loop, and calculated the discrepancy between both signals after each iteration, for more than 100 successive cycles.

\section{Control structure for closed-loop adjustment of EES}

We modeled stepping patterns as a discrete-time, SISO system (stimulation frequency/step height). The continuous flow of kinematic information was discretized by gait cycles using foot strike events, which separated consecutive gait cycles and defined the set of sampling times $K=\left\{k_{1}, k_{2} \ldots k_{n}\right\}$. These events triggered the update of the stimulation frequency, which was applied using zero-order hold control until the next event. The maximum step height during each gait cycle (defined as $h_{i}=\max \left(h_{t}\right), t \in\left[k_{i-1}, k_{i}\right]$ ) served as feedback signal for closed-loop corrections. Stimulation values were set identically for both electrodes and effectively acted as a unique value. The controller used an adaptive input-output forward model built from the linear correlations between stimulation frequency and step height. Regression estimates (slope $\hat{a}_{i}$ and intercept $\hat{b}_{i}$ ) were initially set for each subject based on offline analyses. These values were iteratively updated at every gait cycle using online adaptive filters (least mean squares):

$$
\hat{a}_{i+1}=\hat{a}_{i}+\mu h_{i} e_{i}
$$

where $\mu=0.1$ is the forgetting factor, and $e_{i}$ is the error between recorded and desired outputs at time $i$. This feed-forward control loop enabled to predict and anticipate the necessary changes in stimulation output. It was combined with a PI feedback loop to correct for unmodeled discrepancies. Values for the proportional $\left(k_{\mathrm{p}}\right)$ and integral $\left(k_{\mathrm{i}}\right)$ corrections were set manually for each rat on the first experimental session $\left(k_{\mathrm{p}}=0.2\right.$ to $0.35, k_{\mathrm{i}}=0.1$ to 0.15$)$, and kept constant during the duration of the recordings. The final stimulation frequency was computed on the basis of the combined feedback and feed-forward components to track a user-defined reference value $r_{i+1}$ for the maximum foot height during the next gait cycle:

$$
f_{i+1}=\left(r_{i+1}-\hat{b}_{i+1}\right) / \hat{a}_{i+1}+\left(k_{\mathrm{p}} e_{i}+k_{\mathrm{i}} E_{i}\right)
$$

where $E_{i}$ is the cumulative error at time $i$, and is iteratively calculated as $E_{i}={ }^{i-1} /{ }_{i} E_{i-1}+1 / i e_{i}, i>0$. The reference included a dead band 
( $\mathrm{db}=5 \mathrm{~mm}$ ), which was included above and below the reference value to account for the inherent variability in stepping when calculating tracking errors: $e_{i}=\max \left(0,\left|r_{i}-h_{i}\right|-d b\right)$. Final stimulation values were applied within the range of values efficient to promote functional locomotor movements, spanning from $20 \mathrm{~Hz}$ onset to $95 \mathrm{~Hz}$ saturation (movie S1). The amplitude of the current delivered through each electrode was calibrated at the beginning of each experimental session to obtain optimal facilitation of locomotion at baseline frequency based on visual observation. The real-time platform and control structure are represented and further explained in fig. S4.

\section{Treadmill paradigm}

Rats were tested in the same conditions as training. Serotonin agonists were administered $10 \mathrm{~min}$ before recording onset. Various experimental conditions were tested during each session that lasted $60 \mathrm{~min}$. The first series of experiments evaluated the functional effects of varying amplitude, pulse width, and frequency of EES. For each session, optimal features for the three parameters of EES to promote robust stepping patterns were searched through visual observations. After this preparatory phase, two features were maintained constant, whereas the tested feature was randomly modulated with fixed increments. A minimum of 10 gait successive cycles were recorded for each experimental condition.

The second series of experiments aimed to evaluate the robustness of the developed control policies. Two testing paradigms were designed to challenge real-time control of foot height. During the first paradigm, termed step increment, the reference band was displaced by a fixed increment $(0.5$ to $2.5 \mathrm{~cm})$ or decrement $(0.5$ to $1.5 \mathrm{~cm})$, and maintained at this new reference for six consecutive steps. During the second paradigm, termed triangular waveform, the reference band was gradually displaced by a fixed increment $(0.5$ to $1.5 \mathrm{~cm})$ or decrement $(0.5$ to $2.5 \mathrm{~cm})$ at each step until the maximum $(7.5 \mathrm{~cm})$ or minimum $(3.5 \mathrm{~cm})$ limit of the locomotor system was met. When reaching the limit, the direction of the reference band displacement was inversed (fig. S5).

\section{Locomotor exhaustion}

Kinematic and EMG activities were recorded during continuous stepping on a treadmill until exhaustion of locomotor movements. For each rat, recordings stopped when the animal was not able to move the limb forward; both hindlimbs were dragging along the treadmill belt. On the first day, stepping was enabled by serotonin agonists and continuous EES applied overlying L2 and S1 spinal segments at a fixed frequency of $40 \mathrm{~Hz}$. On the second day, the controller was set to modulate EES frequency to maintain step height within a reference band fixed at $4.5 \mathrm{~cm}$. These experiments took place at 7 weeks post-SCI, when substantial exhaustion of locomotor activity occurs clearly (22). The duration of stepping within the reference band, and until exhaustion of locomotion, was measured for each rat and condition.

\section{Staircase paradigm}

Rats were positioned in a robotic support system (40) that provided a constant force against the direction of gravity (25\% of body weight) while moving the rat forward at a fixed velocity $(9 \mathrm{~cm} / \mathrm{s})$ along a horizontal runway (Fig. 7). Staircases of various heights $(1.3$ to $3.5 \mathrm{~cm})$, lengths (12 to $25 \mathrm{~cm}$ ), and numbers (one or four stairs) were interposed along the runway. For each rat, 100 (single) or 20 (successive) trials were performed along each type of staircase paradigms under noncontrolled or controlled conditions. The percentage of trials dur- ing which the rat tumbled, touched, or passed the entire staircase was evaluated offline using video recordings.

\section{Statistics}

All data are reported as means \pm SEM, unless otherwise specified. Repeated-measures ANOVAs were used to evaluate differences between normally distributed data (Kolmogorov-Smirnov test) from the various experimental conditions. The two-tailed Kruskal-Wallis test was used for nonparametric evaluations. Post hoc differences were assessed using the Bonferroni test. The statistical $\alpha$ level $P<0.05$ was considered significant.

\section{SUPPLEMENTARY MATERIALS}

www.sciencetranslationalmedicine.org/cgi/content/full/6/255/255ra133/DC1 Methods

Fig. S1. Completeness of the SCI.

Fig. S2. Modulation of EES frequency tunes multiple aspects of gait patterns.

Fig. S3. Modulation of EES frequency tunes mono- and polysynaptic responses in flexor and extensor muscle during locomotion.

Fig. S4. Real-time monitoring and control platform.

Fig. S5. High-fidelity control of complex foot trajectory.

Fig. S6. Execution along staircases of various heights and lengths.

Table S1. List of computed kinematic, kinetic, and EMG parameters.

Movie S1. Real-time monitoring and control platform.

Movie S2. Closed-loop neuromodulation achieves high-fidelity control of leg movements.

Movie S3. Closed-loop neuromodulation prevents rapid fatigue during continuous locomotion. Movie S4. Closed-loop neuromodulation enables locomotion across staircases.

\section{REFERENCES AND NOTES}

1. A. M. Lozano, N. Lipsman, Probing and regulating dysfunctional circuits using deep brain stimulation. Neuron 77, 406-424 (2013).

2. D. Borton, S. Micera, R. Millán Jdel, G. Courtine, Personalized neuroprosthetics. Sci. Transl. Med. 5, 210 rv212 (2013).

3. P. Krack, A. Batir, N. Van Blercom, S. Chabardes, V. Fraix, C. Ardouin, A. Koudsie, P. D. Limousin, A. Benazzouz, J. F. LeBas, A. L. Benabid, P. Pollak, Five-year follow-up of bilateral stimulation of the subthalamic nucleus in advanced Parkinson's disease. N. Engl. J. Med. 349, 1925-1934 (2003).

4. B. Rosin, M. Slovik, R. Mitelman, M. Rivlin-Etzion, S. N. Haber, Z. Israel, E. Vaadia, H. Bergman, Closed-loop deep brain stimulation is superior in ameliorating parkinsonism. Neuron $\mathbf{7 2}$, 370-384 (2011).

5. P. A. Tass, L. Qin, C. Hauptmann, S. Dovero, E. Bezard, T. Boraud, W. G. Meissner, Coordinated reset has sustained aftereffects in Parkinsonian monkeys. Ann. Neurol. 72, 816-820 (2012).

6. D. T. Brocker, B. D. Swan, D. A. Turner, R. E. Gross, S. B. Tatter, M. M. Koop, H. Bronte-Stewart, W. M. Grill, Improved efficacy of temporally non-regular deep brain stimulation in Parkinson's disease. Exp. Neurol. 239, 60-67 (2013).

7. R. van den Brand, J. Heutschi, Q. Barraud, J. DiGiovanna, K. Bartholdi, M. Huerlimann, L. Friedli, I. Vollenweider, E. M. Moraud, S. Duis, N. Dominici, S. Micera, P. Musienko, G. Courtine, Restoring voluntary control of locomotion after paralyzing spinal cord injury. Science 336, 1182-1185 (2012).

8. S. Harkema, Y. Gerasimenko, J. Hodes, J. Burdick, C. Angeli, Y. Chen, C. Ferreira, A. Willhite, E. Rejc, R. G. Grossman, V. R. Edgerton, Effect of epidural stimulation of the lumbosacral spinal cord on voluntary movement, standing, and assisted stepping after motor complete paraplegia: A case study. Lancet 377, 1938-1947 (2011).

9. B. J. Holinski, D. G. Everaert, V. K. Mushahwar, R. B. Stein, Real-time control of walking using recordings from dorsal root ganglia. J. Neural Eng. 10, 056008 (2013).

10. R. Fuentes, P. Petersson, W. B. Siesser, M. G. Caron, M. A. Nicolelis, Spinal cord stimulation restores locomotion in animal models of Parkinson's disease. Science 323, 1578-1582 (2009).

11. G. Fénelon, C. Goujon, J. M. Gurruchaga, P. Cesaro, B. Jarraya, S. Palfi, J. P. Lefaucheur, Spinal cord stimulation for chronic pain improved motor function in a patient with Parkinson's disease. Parkinsonism Relat. Disord. 18, 213-214 (2012). 
12. J. T. Paz, T. J. Davidson, E. S. Frechette, B. Delord, I. Parada, K. Peng, K. Deisseroth, J. R. Huguenard, Closed-loop optogenetic control of thalamus as a tool for interrupting seizures after cortical injury. Nat. Neurosci. 16, 64-70 (2013).

13. K. Minassian, B. Jilge, F. Rattay, M. M. Pinter, H. Binder, F. Gerstenbrand, M. R. Dimitrijevic, Stepping-like movements in humans with complete spinal cord injury induced by epidural stimulation of the lumbar cord: Electromyographic study of compound muscle action potentials. Spinal Cord 42, 401-416 (2004).

14. G. Courtine, Y. Gerasimenko, R. van den Brand, A. Yew, P. Musienko, H. Zhong, B. Song, Y. Ao, R. M. Ichiyama, I. Lavrov, R. R. Roy, M. V. Sofroniew, V. R. Edgerton, Transformation of nonfunctional spinal circuits into functional states after the loss of brain input. Nat. Neurosci. 12, 1333-1342 (2009).

15. V. F. Lyalka, L. J. Hsu, A. Karayannidou, P. V. Zelenin, G. N. Orlovsky, T. G. Deliagina, Facilitation of postural limb reflexes in spinal rabbits by serotonergic agonist administration, epidural electrical stimulation, and postural training. J. Neurophysiol. 106, 1341-1354 (2011).

16. P. Afshar, A. Khambhati, S. Stanslaski, D. Carlson, R. Jensen, D. Linde, S. Dani, M. Lazarewicz, P. Cong, J. Giftakis, P. Stypulkowski, T. Denison, A translational platform for prototyping closed-loop neuromodulation systems. Front. Neural Circuits 6, 117 (2013).

17. M. Capogrosso, N. Wenger, S. Raspopovic, P. Musienko, J. Beauparlant, L. Bassi Luciani, G. Courtine, S. Micera, A computational model for epidural electrical stimulation of spinal sensorimotor circuits. J. Neurosci. 33, 19326-19340 (2013).

18. P. Gad, I. Lavrov, P. Shah, H. Zhong, R. R. Roy, V. R. Edgerton, Y. Gerasimenko, Neuromodulation of motor-evoked potentials during stepping in spinal rats. J. Neurophysiol. 110, 1311-1322 (2013).

19. I. Lavrov, C. J. Dy, A. J. Fong, Y. Gerasimenko, G. Courtine, H. Zhong, R. R. Roy, V. R. Edgerton, Epidural stimulation induced modulation of spinal locomotor networks in adult spinal rats. J. Neurosci. 28, 6022-6029 (2008).

20. J. Ladenbauer, K. Minassian, U. S. Hofstoetter, M. R. Dimitrijevic, F. Rattay, Stimulation of the human lumbar spinal cord with implanted and surface electrodes: A computer simulation study. IEEE Trans. Neural Syst. Rehabil. Eng. 18, 637-645 (2010).

21. V. Dietz, R. Müller, Degradation of neuronal function following a spinal cord injury: Mechanisms and countermeasures. Brain 127, 2221-2231 (2004).

22. J. Beauparlant, R. van den Brand, Q. Barraud, L. Friedli, P. Musienko, V. Dietz, G. Courtine, Undirected compensatory plasticity contributes to neuronal dysfunction after severe spinal cord injury. Brain 136, 3347-3361 (2013).

23. C. A. Angeli, V. R. Edgerton, Y. P. Gerasimenko, S. J. Harkema, Altering spinal cord excitability enables voluntary movements after chronic complete paralysis in humans. Brain 137, 1394-1409 (2014).

24. H. Vallery, P. Lutz, J. von Zitzewitz, G. Rauter, M. Fritschi, C. Everarts, R. Ronsse, A. Curt M. Bolliger, S. B. Pai, Multidirectional transparent support for overground gait training, IEEE International Conference on Rehabilitation Robotics (ICORR), Seattle, WA, 24 to 26 June 2013.

25. D. A. McCrea, Spinal circuitry of sensorimotor control of locomotion. J. Physiol. 533, 41-50 (2001).

26. Y. Gerasimenko, R. Gorodnichev, E. Machueva, E. Pivovarova, D. Semyenov, A. Savochin, R. R. Roy, V. R. Edgerton, Novel and direct access to the human locomotor spinal circuitry. J. Neurosci. 30, 3700-3708 (2010).

27. G. Courtine, S. J. Harkema, C. J. Dy, Y. P. Gerasimenko, P. Dyhre-Poulsen, Modulation of multisegmental monosynaptic responses in a variety of leg muscles during walking and running in humans. J. Physiol. 582, 1125-1139 (2007).

28. J. B. Zimmermann, K. Seki, A. Jackson, Reanimating the arm and hand with intraspinal microstimulation. J. Neural Eng. 8, 054001 (2011).

29. D. Kernell, Principles of force gradation in skeletal muscles. Neural Plast. 10, 69-76 (2003).

30. K. Minassian, I. Persy, F. Rattay, M. M. Pinter, H. Kern, M. R. Dimitrijevic, Human lumbar cord circuitries can be activated by extrinsic tonic input to generate locomotor-like activity. Hum. Mov. Sci. 26, 275-295 (2007).

31. Y. Gerasimenko, R. R. Roy, V. R. Edgerton, Epidural stimulation: Comparison of the spinal circuits that generate and control locomotion in rats, cats and humans. Exp. Neurol. 209 417-425 (2008).

32. G. P. Braz, M. Russold, G. M. Davis, Functional electrical stimulation control of standing and stepping after spinal cord injury: A review of technical characteristics. Neuromodulation 12, 180-190 (2009).
33. C. L. Lynch, M. R. Popovic, Functional electrical stimulation. IEEE Contr. Syst. Mag. 28, 40-50 (2008).

34. E. C. Field-Fote, Combined use of body weight support, functional electric stimulation, and treadmill training to improve walking ability in individuals with chronic incomplete spinal cord injury. Arch. Phys. Med. Rehabil. 82, 818-824 (2001).

35. G. Bosco, R. E. Poppele, Proprioception from a spinocerebellar perspective. Physiol. Rev. 81, 539-568 (2001).

36. G. Courtine, R. R. Roy, J. Hodgson, H. McKay, J. Raven, H. Zhong, H. Yang, M. H. Tuszynski, V. R. Edgerton, Kinematic and EMG determinants in quadrupedal locomotion of a non-human primate (Rhesus). J. Neurophysiol. 93, 3127-3145 (2005).

37. Y. P. Ivanenko, A. d'Avella, R. E. Poppele, F. Lacquaniti, On the origin of planar covariation of elevation angles during human locomotion. J. Neurophysiol. 99, 1890-1898 (2008).

38. D. Borton, M. Bonizzato, J. Beauparlant, J. DiGiovanna, E. M. Moraud, N. Wenger, P. Musienko, I. R. Minev, S. P. Lacour, R. Millán Jdel, S. Micera, G. Courtine, Corticospinal neuroprostheses to restore locomotion after spinal cord injury. Neurosci. Res. 78, 21-29 (2014).

39. B. Mariani, S. Rochat, C. J. Büla, K. Aminian, Heel and toe clearance estimation for gait analysis using wireless inertial sensors. IEEE Trans. Biomed. Eng. 59, 3162-3168 (2012).

40. N. Dominici, U. Keller, H. Vallery, L. Friedli, R. van den Brand, M. L. Starkey, P. Musienko, R. Riener, G. Courtine, Versatile robotic interface to evaluate, enable and train locomotion and balance after neuromotor disorders. Nat. Med. 18, 1142-1147 (2012).

41. V. R. Edgerton, G. Courtine, Y. P. Gerasimenko, I. Lavrov, R. M. Ichiyama, A. J. Fong, L. L. Cai, C. K. Otoshi, N. J. K. Tillakaratne, J. W. Burdick, R. R. Roy, Training locomotor networks. Brain Res. Rev. 57, 241-254 (2008).

42. P. Musienko, R. van den Brand, O. Märzendorfer, R. R. Roy, Y. Gerasimenko, V. R. Edgerton, G. Courtine, Controlling specific locomotor behaviors through multidimensional monoaminergic modulation of spinal circuitries. J. Neurosci. 31, 9264-9278 (2011).

43. I. Steuer, P. Rouleau, P. A. Guertin, Pharmacological approaches to chronic spinal cord injury. Curr. Pharm. Des. 19, 4423-4436 (2013).

44. C. Ethier, E. R. Oby, M. J. Bauman, L. E. Miller, Restoration of grasp following paralysis through brain-controlled stimulation of muscles. Nature 485, 368-371 (2012).

Acknowledgments: We thank D. Borton for helpful advice on the manuscript, S. Duis and K. Bartholdi for help with animal care, A. Vuaridel for data processing, and J. Gandar and A. Kuck for their contribution to real-time platform development. Funding: A Starting Grant from the European Research Council (ERC 261247, Walk Again), the European Community's Seventh Framework Program (CP-IP 258654, NeuWALK), the International Paraplegic Foundation, the Bertarelli Foundation, and funding from the National Center of Competence in Research (NCCR) in Robotics and NanoTera.ch program of the Swiss National Science Foundation (SpineRepair). Author contributions: N.W., E.M.M., S.R., and M.B. performed the in vivo experiments. N.W., E.M.M., and G.C. analyzed the data. N.W. and E.M.M. conducted the statistical analysis. P.M. implanted the epidural electrodes and placed the SCl, whereas N.W. and G.C. inserted the EMG electrodes. N.W. E.M.M., J.D., M.M., S.M., and G.C. conceived the experiments. N.W., E.M.M., and G.C. prepared the figures with the help of the other authors. N.W., E.M.M., and G.C. wrote the manuscript, and all the authors contributed to its editing. G.C. supervised all aspects of the work. Competing interests: Ongoing European patent application (no. 13191003.6) on the stimulation algorithms. The authors declare no other competing interests. Data and materials availability: Raw data and customdeveloped $\mathrm{C}++$ and TDT (Tucker-Davis Technologies) codes will be made available through material transfer agreement upon request to G.C.

Submitted 18 December 2013

Accepted 5 May 2014

Published 24 September 2014

$10.1126 /$ scitranslmed.3008325

Citation: N. Wenger, E. M. Moraud, S. Raspopovic, M. Bonizzato, J. DiGiovanna, P. Musienko, M. Morari, S. Micera, G. Courtine, Closed-loop neuromodulation of spinal sensorimotor circuits controls refined locomotion after complete spinal cord injury. Sci. Transl. Med. 6, 255ra133 (2014). 